\title{
Adaptive Droop Coefficient and SOC Equalization-Based Primary Frequency Modulation Control Strategy of Energy Storage
}

\author{
Gaojun Meng ${ }^{1}$, Yang Lu ${ }^{1}$, Haitao Liu ${ }^{1}$, Yuan Ye ${ }^{2, *}$, Yukun Sun ${ }^{1}$ and Wenyi Tan ${ }^{1}$ \\ 1 School of Electric Power Engineering, Nanjing Institute of Technology, Nanjing 211167, China; \\ gjun_m@126.com (G.M.); luyang@126.com (Y.L.); liuhaitao@126.com (H.L.); sunyukun@126.com (Y.S.); \\ tanwenyi@126.com (W.T.) \\ 2 School of Electrical and Information Engineering, Jiangsu University, Zhenjiang 212013, China \\ * Correspondence: 1000050003@ujs.edu.cn
}

Citation: Meng, G.; Lu, Y.; Liu, H.; Ye, Y.; Sun, Y.; Tan, W. Adaptive Droop Coefficient and SOC

Equalization-Based Primary Frequency Modulation Control Strategy of Energy Storage. Electronics 2021, 10, 2645. https://doi.org/ 10.3390/electronics10212645

Academic Editor: Noel Rodriguez

Received: 25 September 2021

Accepted: 22 October 2021

Published: 29 October 2021

Publisher's Note: MDPI stays neutral with regard to jurisdictional claims in published maps and institutional affiliations.

Copyright: (c) 2021 by the authors. Licensee MDPI, Basel, Switzerland. This article is an open access article distributed under the terms and conditions of the Creative Commons Attribution (CC BY) license (https:/ / creativecommons.org/licenses/by/ $4.0 /)$.

\begin{abstract}
In order to efficiently use energy storage resources while meeting the power grid primary frequency modulation requirements, an adaptive droop coefficient and SOC balance-based primary frequency modulation control strategy for energy storage is proposed. Taking the SOC of energy storage battery as the control quantity, the depth of energy storage output is adaptively adjusted to prevent the saturation or exhaustion of energy storage SOC. The balanced control strategy is introduced to realize the rational utilization of resources and the fast balance of SOC in the process of primary frequency modulation of energy storage battery under different charge states. Then, four evaluation indexes are proposed to evaluate the effect of primary frequency modulation and SOC maintenance. Taking a regional power grid as an example, a simulation analysis is carried out under step load disturbance and continuous load disturbance. According to the simulation results, the proposed control strategy is effective in power system frequency regulation and battery SOC maintenance.
\end{abstract}

Keywords: battery energy storage system; primary frequency modulation; state of charge; droop control; equalization strategy

\section{Introduction}

In recent years, new energy power generation has developed rapidly. By the end of 2019 , the total installed capacity of wind power in China reached $210 \mathrm{GW}$, and the total installed photovoltaic capacity reached 204 GW [1]. Especially in many provinces of the "three northern regions", the proportion of wind power installed in the provincial power grid reached more than $40 \%$. However, the wind turbine itself is decoupled from the frequency of the power system, and the replacement of traditional units by high-proportion wind power will lead to the weakening of system inertia and the reduction in primary frequency modulation capacity [2]. In addition, the randomness and volatility of new energy generation become more prominent when it is connected to the grid on a large scale, which will bring greater power disturbance to the power grid [3-6]. This suggests that under such dual pressure, there is an urgent need to make up for the lack of primary frequency modulation capacity in the traditional way with the help of new frequency modulation means.

In recent years, electrochemical energy storage has been widely used in the field of power grid auxiliary frequency modulation because of its advantages, such as rapid action and flexible control. At present, the total number of global megawatt electrochemical energy storage and frequency modulation projects has reached more than $160[7,8]$, but the installed capacity is only $2457 \mathrm{MW}$. Comparatively speaking, the energy storage capacity only accounts for a very small part of the power grid capacity, so how to use the energy 
storage with limited capacity to safely and continuously assist the primary frequency modulation puts forward higher requirements for the scientific and efficient control strategy. It is worth noting that scholars at home and abroad have carried out a series of research work in the field of energy storage control strategy [9].

Reference [10] puts forward a droop control strategy for energy storage battery participating in power grid frequency regulation. By adjusting the output of the energy storage battery according to a fixed sagging coefficient, the power can be adjusted quickly and has a better frequency modulation effect. In reference [11], an energy storage battery based on virtual droop control is reported to solve the power grid frequency stability, and the steady-state effect of the droop control strategy is verified. However, the proposed control strategies do not take into account the changes and limitations of the dynamic characteristics of the system, the frequency modulation requirements, and the SOC of the energy storage battery. The maintenance of energy storage SOC is the key to the efficient utilization of its electricity [12]. Some studies have taken into account the problem of SOC management in the process of energy storage participating in FM. In reference [13], the control strategy of energy storage white adaptive frequency modulation and self-recovery is expounded by using the smoothing characteristic of logistic regression function. Although the SOC of energy storage battery is considered in reference [14], the regulation strategy of fixed power output participating in power grid frequency is still adopted. Reference [15] provides an adaptive control strategy for energy storage. When the electric vehicle is connected to the grid to charge, on the premise of meeting the charging needs of users, the frequency adjustment service can be provided flexibly for the power grid according to the power status and SOC status. Unfortunately, its research content is mainly aimed at the decentralized energy storage battery for electric vehicle charging needs, but how to give full play to its auxiliary grid frequency modulation performance according to the SOC characteristics of centralized energy storage battery remains a significant challenge.

In order to ensure the effect of frequency modulation while ensuring the state of energy storage SOC and maintaining the long-term stable output of energy storage, an adaptive primary frequency modulation control strategy considering SOC feedback of energy storage battery is proposed in this paper. Taking the SOC of the energy storage battery as the control quantity, the adaptive output law of the energy storage unit is designed, the output adjustment of the energy storage unit under different frequency deviation and SOC interval is optimized, and the balanced control strategy is introduced to realize the rational utilization of resources and the fast balance of SOC in the primary frequency modulation process of the energy storage battery under different charge states. Finally, taking the regional power grid as an example, the effectiveness of the proposed control strategy is verified by simulation under the condition of step load disturbance and continuous load disturbance.

\section{Primary Frequency Modulation Control Mode of Regional Power Grid with Energy Storage}

Virtual droop control and virtual inertial control are classical control strategies applied to energy storage and frequency modulation [16]. The virtual inertial control can quickly restrain the initial frequency change rate but has no effect on the steady-state deviation; the virtual droop control can improve the steady-state deviation but has no effect on the initial frequency difference change rate. Therefore, in practical application, we can combine the characteristics of the two by determining the appropriate timing of control to achieve complementary advantages.

In this paper, the virtual droop control is used as the main control of the battery energy storage to participate in the primary frequency modulation. As long as the frequency deviation appears, the energy storage battery can change its output under the virtual droop control and automatically respond to the frequency change of the system. Its control function runs through the whole process of primary frequency modulation. As a supplementary control of droop control, virtual inertial control only acts at the initial 
time of frequency change, restrains the speed of frequency deterioration, and increases the inertia of the system. The timing of its control can be expressed by Equation (1).

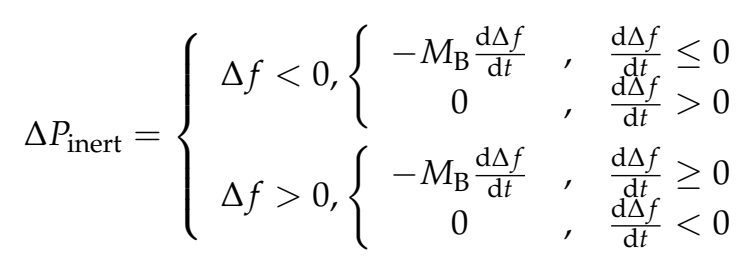

where $\Delta f$ represents the frequency deviation of the power network, MB represents the virtual inertia coefficient, and $\mathrm{d} \Delta f / \mathrm{d} t$ represents the frequency change rate.

As a result, an energy storage primary frequency modulation droop control strategy based on inertial response is constructed, and the reference output power expression of the energy storage unit is shown in Equation (2).

$$
\Delta P_{\mathrm{B}}=-\left(K_{\mathrm{B}} \Delta f+M_{\mathrm{B}} \frac{\mathrm{d} \Delta f}{\mathrm{~d} t}\right)
$$

where $K_{\mathrm{B}}$ represents the droop control coefficient, $\Delta f$ represents grid frequency deviation, $\mathrm{MB}$ represents the virtual inertia coefficient, and $\mathrm{d} \Delta f / \mathrm{d} t$ represents the frequency change rate.

Figure 1 shows the flow chart of the integrated control mode adopted in this paper. As an inertial response link, virtual inertial control plays a role in the frequency deterioration stage and is removed in the recovery phase. The energy storage unit always provides power support for the system through droop control until the frequency is restored to the specified dead partition of the grid frequency.

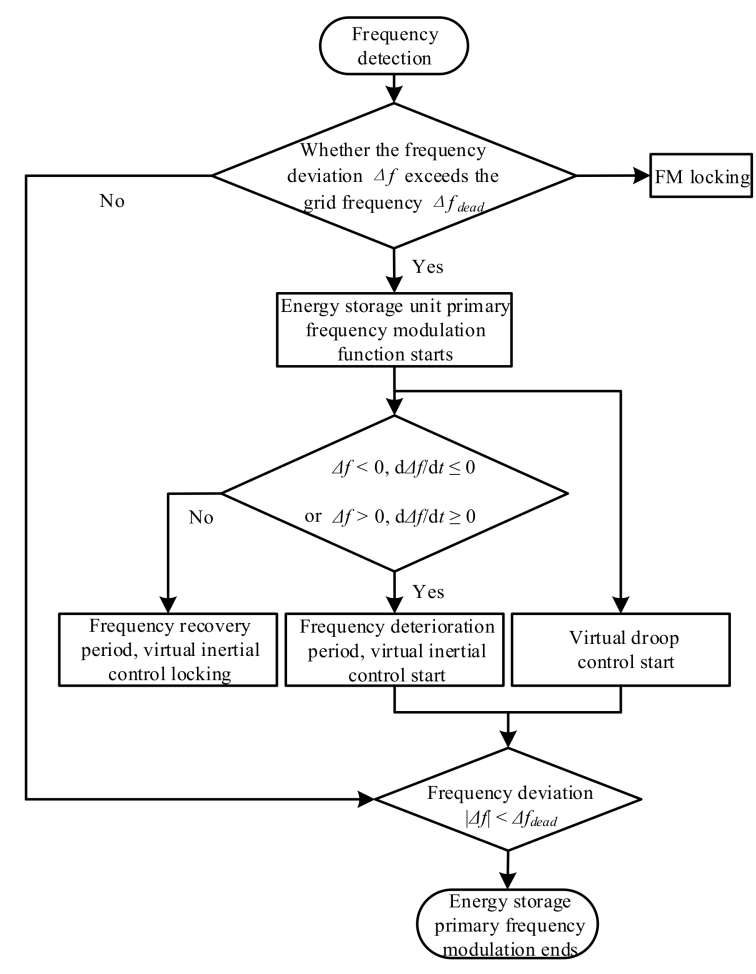

Figure 1. Process of control model.

\section{Primary Frequency Modulation Control Strategy of Energy Storage}

At present, in the traditional droop control based on virtual inertia, the fixed unit power value $K_{\max }$ is usually used to participate in the primary frequency modulation. As the current energy storage capacity is still very limited, compared with the large power 
grid, with the long-term use of $K_{\max }$ to charge and discharge the power grid, in the process of frequent operation, it is easy to cause the battery to be in the state of overcharge and overdischarge for a long time [17].

In addition, the large-capacity energy storage system is usually composed of multiple energy storage units. Due to the influence of battery manufacturing technology and initial electricity in each energy storage unit, there is a problem of imbalance of SOC in the energy storage unit. In the actual process of participating in frequency modulation, each energy storage unit adopts a unified droop control mode for charge and discharge. Then, the energy storage unit with lower SOC in discharge mode or higher SOC in charging mode will withdraw from the frequency modulation process ahead of time, and the remaining energy storage unit must be forced to overcharge and overdischarge, which will shorten the service life of some energy storage units in the system [18].

Based on this, we propose a SOC feedback-based improved droop control strategy, which adaptively adjusts the power coefficient of the droop control unit and introduces an equalization factor to coordinate and optimize the power output of each energy storage unit so as to improve the overall performance of the energy storage system participating in the frequency modulation of the power system.

\subsection{BESS Adaptive Droop Coefficient Setting}

In order to prevent the greater impact on the power grid frequency caused by the saturation or exhaustion of the energy storage SOC, we adopt the method of adaptively adjusting the virtual droop control coefficient $K_{\mathrm{B}}$ according to the real-time state of the energy storage SOC, establish a piecewise function with the energy storage battery SOC as the independent variable and the droop control coefficient $K_{\mathrm{B}}$ as the dependent variable, and design the adaptive output law of energy storage charge and discharge.

As shown in Figure 2, the battery power is divided into seven SOC intervals, and the adaptive dynamic adjustment is carried out in each interval. The optimization of the unit regulation power coefficient of the droop control of the energy storage unit is more detailed and targeted, and the specific ways are as follows.

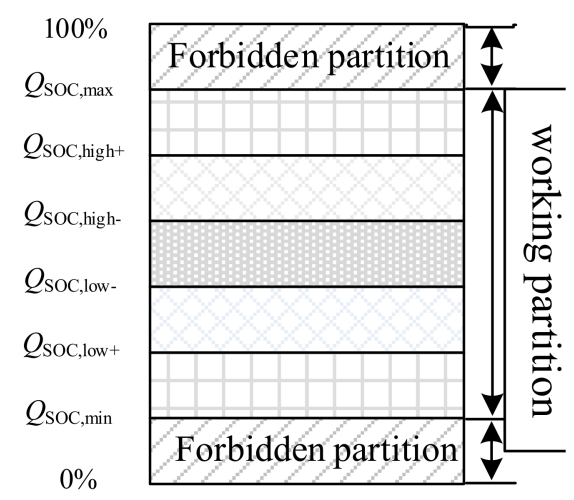

Figure 2. Schematic of energy storage battery SOC partition.

(1) $Q_{\mathrm{SOC}}(\mathrm{t}) \in\left[0 \%, Q_{\mathrm{SOC}, \min }\right]$ or $\left[Q_{\mathrm{SOC}, \max }, 100 \%\right]$

In terms of battery life, deep charge and discharge will accelerate the decline of battery performance of the energy storage unit. Therefore, in actual operation, the energy storage SOC is usually required to be kept within a reasonable range, that is, the following:

$$
\Delta P_{\mathrm{B}}=-\left(K_{\mathrm{B}} \Delta f+M_{\mathrm{B}} \frac{\mathrm{d} \Delta f}{\mathrm{~d} t}\right)
$$

When the BMS of the energy storage unit detects that the real-time value of the battery's SOC $Q_{\mathrm{SOC}}(t)$ has crossed the reasonable range, that is, $Q_{\mathrm{SOC}}(t)>Q_{\mathrm{SOC} \text {, max }}$ or $Q_{\text {SOC }}(t)<Q_{\text {SOC,min }}$ (this article is set as the battery forbidden interval), the energy storage 
stops output to avoid affecting its own battery life. Usually, take $Q_{S O C, \max }=0.9$, and $Q_{\text {SOC, } \min }=0.1[19,20]$.

(2) $Q_{\text {soc }}(t) \in\left[Q_{\text {soc }, \text { low- }}, Q_{\text {soc,high- }}\right]$

In this range, the energy storage capacity is relatively sufficient. The expected value of $50 \%$ of the battery SOC is within this range, and the energy storage unit has sufficient bidirectional frequency modulation ability to cope with the increase or decrease in frequency. Whether charging or discharging, the energy storage unit uses the maximum droop control unit to adjust the power coefficient $K_{\max }$.

$$
\begin{aligned}
& K_{\mathrm{B}}=\left\{\begin{array}{l}
K_{\mathrm{ch}}=K_{\max } \\
K_{\text {disch }}=K_{\max }
\end{array}\right. \\
& \quad, Q_{\text {SOC }}(t) \in\left[Q_{\text {SOC,low }-}, Q_{\text {SOC,high- }}\right]
\end{aligned}
$$

(3) $Q_{\text {sOC }}(t) \in\left[Q_{\text {sOC,min }}, Q_{\text {sOC,low- }}\right]$

In this range, the energy storage unit has sufficient charging capacity and insufficient discharge capacity. Thus, in the charging mode, the energy storage unit adjusts the power coefficient $K_{\max }$ with the maximum unit to output.

$$
K_{\mathrm{B}}=K_{\mathrm{ch}}=K_{\max }, Q_{\mathrm{SOC}}(t) \in\left[Q_{\mathrm{sOC}, \min ,} Q_{\mathrm{sOC}, \mathrm{low}_{-}}\right)
$$

In the discharge mode, the unit regulation power coefficient $K_{\mathrm{B}}$ is adaptively adjusted according to the SOC of the energy storage battery, and the specific value can be determined by Equations (6) and (7).

When $\mathrm{QSOC}_{\mathrm{SO}}(t) \in\left[\mathrm{Q}_{\mathrm{SOC}, \min }, \mathrm{Q}_{\mathrm{SOC}, \mathrm{low}_{+}}\right]$, we have the following:

$$
K_{\mathrm{B}}=K_{\text {disch }}=\frac{1}{2} K_{\max }\left(1-\sqrt{\frac{Q_{\mathrm{SOC}}(t)-Q_{\mathrm{SOC}, \text { low }+}}{Q_{\mathrm{SOC}, \min }-Q_{\mathrm{SOC}, \mathrm{low}+}}}\right)
$$

When $\mathrm{QSOC}_{\mathrm{SOC}}(t) \in\left(\mathrm{Q}_{\mathrm{SOC}, \text { low }^{+}}, \mathrm{Q}_{\mathrm{SOC}, \text { low- }}\right)$,

$$
K_{\mathrm{B}}=K_{\text {disch }}=\frac{1}{2} K_{\max }\left(1-\sqrt{\frac{Q_{\mathrm{SOC}}(t)-Q_{\mathrm{SOC}, \mathrm{low}-}}{Q_{\mathrm{SOC}, \text { low }+}-Q_{\mathrm{SOC}, \mathrm{low}-}}}\right)
$$

(4) $\mathrm{Q}_{\mathrm{SOC}}(t) \in\left(\mathrm{Q}_{\mathrm{SOC}, \text { high-}}, \mathrm{Q}_{\mathrm{sOC}, \mathrm{max}}\right]$

In this range, the energy storage unit has sufficient discharge capacity and insufficient charging capacity. So, in the charging mode, the $K_{B}$ needs to be adaptively adjusted, and its values are shown in Equations (8) and (9).

When $\mathrm{Q}_{\mathrm{SOC}}(t) \in\left[\mathrm{Q}_{\mathrm{SOC}, \text { high+ }}, \mathrm{Q}_{\mathrm{SOC}, \max }\right]$, then we have the following:

$$
K_{\mathrm{B}}=K_{\mathrm{ch}}=\frac{1}{2} K_{\max }\left(1-\sqrt{\frac{Q_{\mathrm{SOC}}(t)-Q_{\mathrm{SOC}, \mathrm{high}+}}{Q_{\mathrm{SOC}, \max }-Q_{\mathrm{SOC}, \mathrm{high}+}}}\right)
$$

When $\mathrm{Q}_{\mathrm{SOC}}\left({ }_{t}\right) \in\left(\mathrm{Q}_{\mathrm{SOC}, \text { high-}}, \mathrm{Q}_{\mathrm{SOC}}\right.$, high+$\left.{ }^{+}\right)$, then we have the following:

$$
K_{\mathrm{B}}=K_{\mathrm{ch}}=\frac{1}{2} K_{\max }\left(1+\sqrt{\frac{Q_{\mathrm{SOC}}(t)-Q_{\mathrm{SOC}, \text { high }+}}{Q_{\mathrm{SOC}, \text { high }-}-Q_{\mathrm{SOC}, \text { high }+}}}\right)
$$

In the discharging mode, the energy storage unit adjusts the power coefficient $K_{\max }$ in the largest unit for output.

$$
\begin{aligned}
& K_{\mathrm{B}}=K_{\mathrm{disch}}=K_{\max } \\
& \quad, Q_{\mathrm{SOC}}(t) \in\left(Q_{\mathrm{SOC}, \text { high }-}, Q_{\mathrm{SOC}, \max }\right)
\end{aligned}
$$




\subsection{BESS Internal Unit SOC Balance}

In addition, in response to the problem of SOC imbalance in the charge and discharge process of the energy storage unit participating in the primary frequency modulation in the system, we introduce the equalization factor $H$ on the basis of the adaptive optimization of the power coefficient $K_{\mathrm{B}}$ of the droop control unit, amplify the difference between the SOC of each unit, adjust the output rate of the battery in different states, change the power responsibility of each unit in the primary frequency modulation, and realize the fast equalization control of the SOC of the energy storage unit.

The equilibrium factor $H$ is added to the droop control in a multiplicative relationship, namely, as follows:

$$
-\left(M_{\mathrm{B}} \frac{\mathrm{d} \Delta f}{\mathrm{~d} t}+K_{\mathrm{B}} \Delta f \cdot H\right)=\Delta P_{\mathrm{B}}
$$

The equilibrium factor $H$ is expressed as follows:

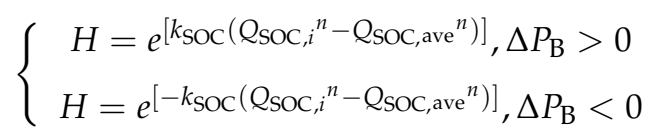

where $Q_{\mathrm{SOC}, i}$ represents the SOC value of each energy storage; $Q_{\mathrm{SOC}, \text { ave }}$ represents the average state of charge of all energy storage units participating in a frequency modulation in the system; $n$ represents the secondary power; and $k_{\mathrm{SOC}}$ represents the state-of-charge adjustment coefficient, which is used to control the equalization speed.

According to Equation (12), under the discharge condition, for the energy storage unit with higher SOC, the equalization factor $H$ is larger, which accelerates the discharge speed of the energy storage battery and releases more electricity. For the energy storage unit with lower SOC, the equilibrium factor $H$ is smaller, which can decelerate and release less electricity. Under the charging condition, the effect is reversed. In addition, in order to maintain the stability of the system, the $K_{\mathrm{B}}$ under the secondary modification of the equilibrium factor cannot exceed its upper and lower limits.

The equalization factor plays a positive role in many aspects; for example, it reasonably allocates the frequency modulation demand of the energy storage unit participating in the primary frequency modulation, effectively prevents some energy storage unit from withdrawing frequency modulation ahead of time due to the lack of SOC state, increases the frequency modulation pressure to the remaining energy storage unit, effectively suppresses the overcharge and overdischarge of part of the energy storage, and promotes the selfrecovery stage of the energy storage battery, which realizes the SOC equilibrium of each energy storage unit in the battery energy storage system, thereby improving the utilization efficiency of the energy storage system.

\subsection{BESS Primary Frequency Modulation Integrated Control Strategy}

Based on the above analysis, on the basis of the determined control mode, combined with the adaptive droop force law and the balanced control among the units, a comprehensive adaptive control strategy for energy storage to participate in the primary frequency modulation of the power grid is formed. The control block diagram is shown in Figure 3.

The operation process is as follows:

(1) First of all, the frequency detection device configured by each unit in the energy storage system monitors and detects the power grid frequency. When the frequency deviation $\Delta f$ is generated, it is further judged whether to cross the frequency regulation dead partition. If the frequency falls beyond the dead partition, the primary FM function is activated; otherwise, each unit locks the function.

(2) When $|\Delta f|>0.033 \mathrm{~Hz}$, each energy storage unit is prepared to output power according to the preset control mode: based on the droop control of the inertia response link. At the same time, a comprehensive study is made on the operation state of the power grid and the SOC state of its own battery. The virtual inertia coefficient $M_{\mathrm{B}}$ is 
determined, the $K_{\mathrm{B}}$ is adaptively adjusted, and the output depth is optimized for the first time.

(3) At the system level, the energy storage power station receives the real-time value of each unit BMS uploaded to SOC, calculates the system $Q_{S O C}$,ave, sends it to each unit, judges the equalization link and calculates the equalization factor by each unit, and modifies the droop control coefficient twice so as to realize the reasonable division of the primary frequency modulation responsibility of each unit.

(4) The energy storage unit outputs power according to the optimized control strategy and judges the change of the power grid frequency until the frequency enters the primary frequency modulation dead partition; thus, the control and optimization process ends.

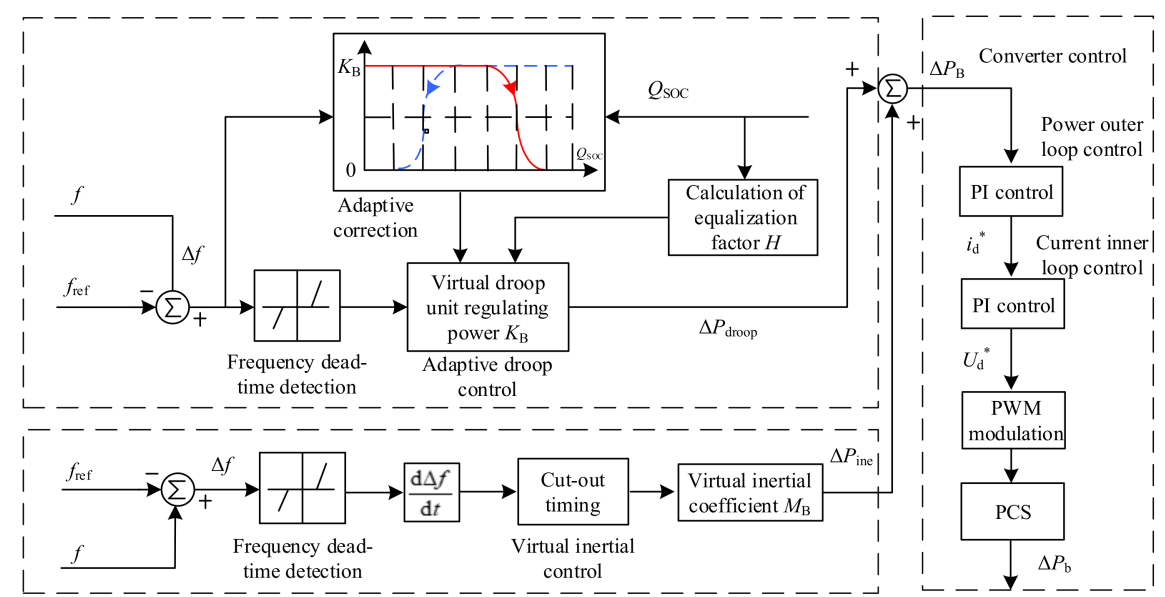

Figure 3. Primary frequency modulation comprehensive adaptive control strategy of BESS.

\section{Energy Storage Frequency Modulation Evaluation Index}

In order to quantitatively evaluate the frequency modulation effect and SOC equalization effect of the proposed strategy, the following evaluation indicators are proposed.

(1) Maximum frequency deviation $\left|\Delta f_{\max }\right|$ : for step load disturbance, corresponding to the maximum frequency deviation after the disturbance, the smaller the $\left|\Delta f_{\max }\right|$, the better the frequency modulation effect.

(2) Frequency deterioration rate $V_{\mathrm{m}}$ : for step load disturbance, it reflects the resistance effect of the system to the sudden change of load. The smaller the $V_{\mathrm{m}}$, the stronger the resistance effect of the system to the sudden change of load, and the better the frequency stability.

$$
V_{\mathrm{m}}=\frac{\left|\Delta f_{\max }\right|}{\Delta t}=\frac{\left|\Delta f_{\max }\right|}{t_{\mathrm{m}}-t_{0}}
$$

where $t_{\mathrm{m}}$ represents the time corresponding to the maximum frequency deviation, and $t_{0}$ represents the time when the load disturbance occurs.

(3) Frequency offset degree $R_{m s}(f)$ : for continuous load disturbance, it reflects the degree to which the system frequency deviates from the standard frequency.

$$
\operatorname{Rms}(f)=\sqrt{\frac{1}{n} \sum_{i=1}^{n}\left(f_{i}-f_{\mathrm{N}}\right)^{2}}
$$

(4) SOC offset $R_{m s}\left(Q_{\mathrm{SOC}}\right)$ : reflects the degree to which SOC deviates from the standard SOC.

$$
\operatorname{Rms}\left(Q_{\mathrm{SOC}}\right)=\sqrt{\frac{1}{n} \sum_{i=1}^{n}\left(Q_{\mathrm{SOC}, i}-Q_{\mathrm{SOC}, \mathrm{N}}\right)^{2}}
$$

where $f i$ and $Q_{\mathrm{SOC}, i}$ represent the frequency and charge state corresponding to the sampling point $i$, respectively; $f_{\mathrm{N}}$ represents the standard frequency of $50 \mathrm{~Hz} ; \mathrm{Q}_{\mathrm{SOC}, \mathrm{N}}$ represents the 
standard SOC, which is 0.5 ; and $n$ represents the total number of sampling points. The smaller the frequency offset and SOC offset is, the better the frequency or SOC deviation is, and the better the FM effect or SOC maintenance effect is.

\section{Calculation Example Analysis}

\subsection{Calculation Example Conditions}

This paper studies a regional power grid, and its primary frequency modulation Matlab/Simulink simulation model is shown in Figure 4. The unit capacity of the power grid is $100 \mathrm{MW}$, the maximum load is $80 \mathrm{MW}$, there are two energy storage sub-units, and their energy storage capacity is $1 \mathrm{MW} / 0.25 \mathrm{MWh}$. The parameters are standardized with the rated frequency and the rated capacity of the unit as the reference value. The maximum unit regulating power $K_{\max }$ of energy storage is 12 , and the unit regulating power $\mathrm{KG}$ of the unit is 20. TG, TCH, TRH and FHP are the governor time constant, steam turbine time constant, reheater time constant and reheater gain, respectively. The values of $0.08 \mathrm{~s}, 0.3 \mathrm{~s}$, $10 \mathrm{~s}$ and 0.5 th $\mathrm{M}$ and $\mathrm{D}$ are the grid inertia time constant and load damping coefficient, respectively. The unit values are 10 and 1 , respectively.

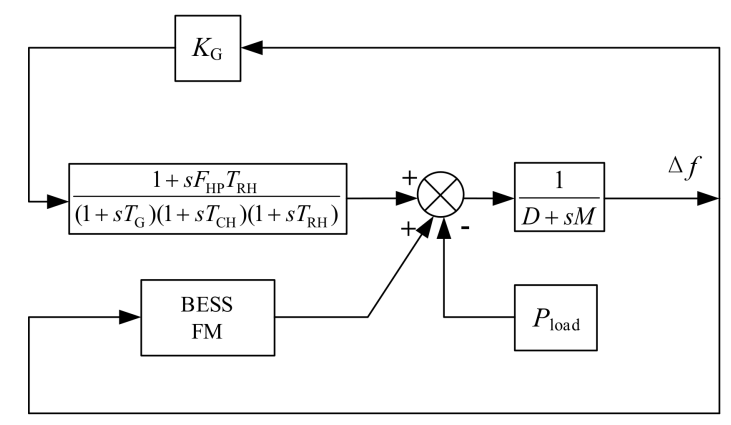

Figure 4. Schematic diagram of primary frequency modulation simulation model.

\subsection{Analysis of Typical Working Conditions}

Under two typical working conditions, the frequency modulation effects of several different control strategies, such as no energy storage, fixed $K$ method, variable $K$ method and this method, are simulated and analyzed.

\subsubsection{Working Condition 1: Step Load Disturbance}

The system is initially stable, the step load disturbance with amplitude of $0.06 \mathrm{pu}$ is added at $10 \mathrm{~s}$, the secondary frequency modulation is not operated, and the simulation time is $150 \mathrm{~s}$. The frequency response and average SOC change curves under several strategies are shown in Figure 5, and the relevant FM indicators are shown in Table 1.

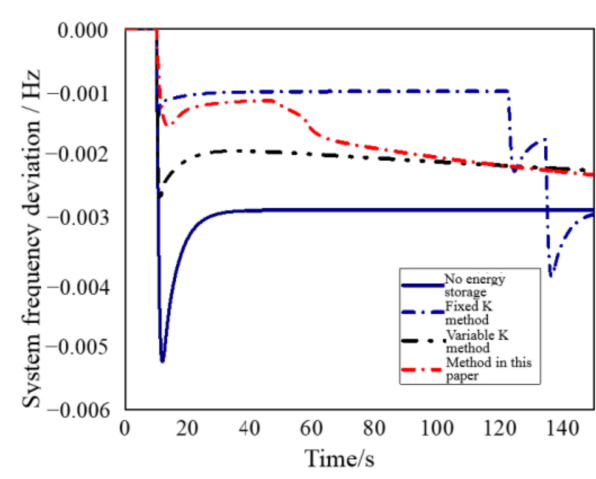

(a)

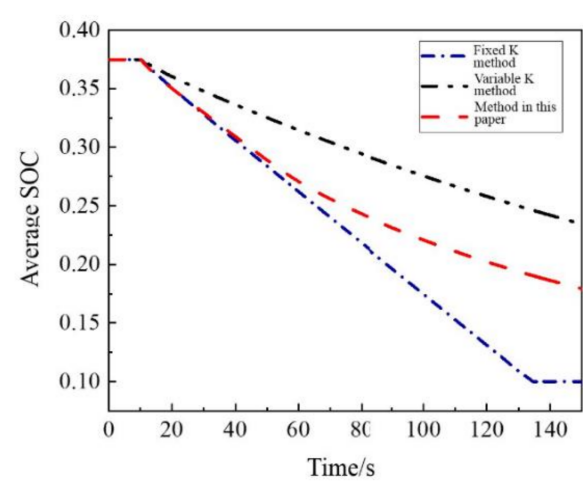

(b)

Figure 5. Response curve of frequency and SOC: (a) frequency response curve; (b) average SOC change curve. 
Table 1. Case 1: indicator statistics of frequency regulation.

\begin{tabular}{ccccc}
\hline & \multicolumn{4}{c}{ FM Method } \\
\cline { 2 - 5 } Indicators & $\begin{array}{c}\text { No Energy } \\
\text { Storage }\end{array}$ & $\begin{array}{c}\text { Variable K } \\
\text { Method }\end{array}$ & Fixed K Method & $\begin{array}{c}\text { Method of This } \\
\text { Study }\end{array}$ \\
\hline$\left|\Delta f_{\max }\right| / 10^{-3}$ & 5.248 & 2.737 & 1.356 & 1.534 \\
$V_{\mathrm{m}} / 10^{-3}$ & 4.006 & 2.607 & 1.067 & 0.410 \\
\hline
\end{tabular}

Comprehensively, in Figure 5a,b, in the initial stage of step disturbance access, the corresponding frequencies of the four frequency modulation modes all decrease rapidly, and the frequency deviation in the case of no energy storage is obviously higher than that of several control modes with energy storage, indicating that the introduction of energy storage can effectively improve the effect of the frequency response. The frequency response effect of the fixed $\mathrm{K}$ method is the best, but the SOC of the energy storage itself is not taken into account. At $134 \mathrm{~s}$, the energy storage reaches the lower limit of energy and cannot continue to modulate frequency; its sudden withdrawal leads to the frequency falling at a larger rate. The SOC maintenance effect of the variable droop coefficient method is the best, but the suppression effect on the frequency change is poor, and the transient frequency drop is larger. This method comprehensively considers the primary frequency modulation requirements of the system and the need to maintain the SOC of the energy storage itself, and plays an effective role in restraining the frequency failure in the initial stage. With the recovery of the frequency of the system, the energy storage enters its own charged state, which makes its own SOC at a high level. According to Table 1, the frequency deterioration rate of this method is obviously lower than that of other energy storage methods.

In this paper, the comprehensive control method of equilibrium factor is introduced, and the SOC changes of the fixed $\mathrm{K}$ method and variable $\mathrm{K}$ method without an equilibrium factor are shown in Figure 6.

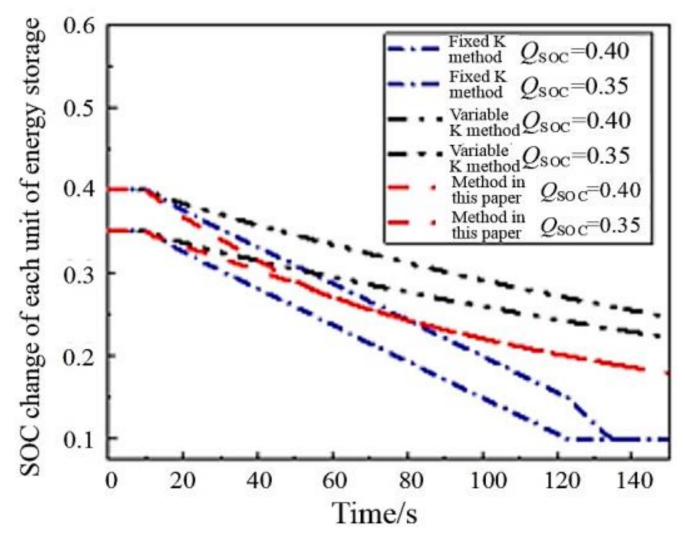

Figure 6. SOC changes with and without equilibrium.

In the aspect of $Q_{\mathrm{SOC}}$ change, in the energy storage system with equilibrium factor, the energy storage unit with higher level of $Q_{\mathrm{SOC}}$ has more output, and the charge state decreases rapidly. The energy storage unit whose $Q_{\mathrm{SOC}}$ is at a lower level has less output, and the charge state decreases slowly. The two tend to be consistent gradually, which not only effectively maintains the SOC level of the energy storage unit in the low charge state, but also avoids the completion of the discharge ahead of time, withdraws from the frequency modulation, resulting in a sudden drop in frequency, or causes the excess output of the residual energy storage, which affects the energy storage life and gives full play to the working efficiency of the energy storage unit in good condition. 


\subsubsection{Working Condition 2: Continuous Load Disturbance}

In order to verify the superiority of this method under continuous load disturbance, 5 min continuous load disturbance is added to the system, and the load disturbance curve is shown in Figure 7.

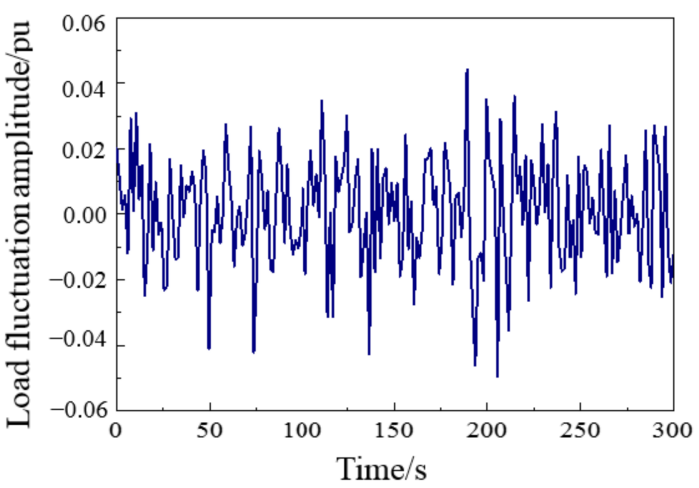

Figure 7. The 5 min load disturbance curve.

From the frequency response curve of Figure 8a and the frequency index data of each method in Table 2, it can be seen that the amplitude of frequency fluctuation of primary frequency modulation with energy storage is much smaller than that without energy storage under continuous load disturbance. Comparing the simulation curves of the three methods with energy storage, we observe that because the unit adjusting power is only $0.5 K_{\max }$ near the $Q_{\mathrm{SOC}}=0.5$, the frequency deviation of the variable $K$ method is the biggest, which cannot give full play to the primary frequency modulation ability of energy storage, cannot effectively cope with the demand of frequency adjustment, and its flexibility is not good. If the same effect as the other two methods is to be achieved, the capacity configuration needs to be increased. The primary frequency modulation effect of the fixed $\mathrm{K}$ method is better, and the frequency deviation can be limited to a small range. In this paper, the frequency modulation effect of the control method is the best, and the designed adaptive droop control output law can make the energy storage unit give full play to the primary frequency modulation ability. In addition, the existence of the virtual inertial control link reduces the change rate of frequency deviation, which makes the frequency suppression effect of this method the best.

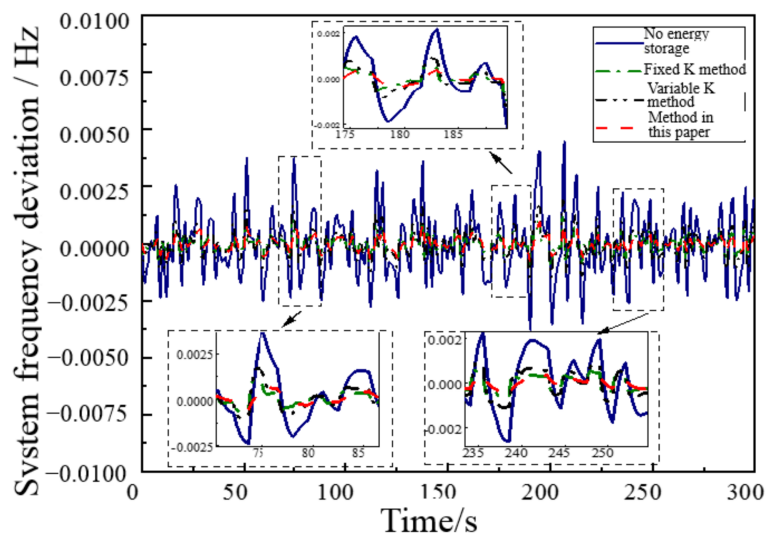

(a)

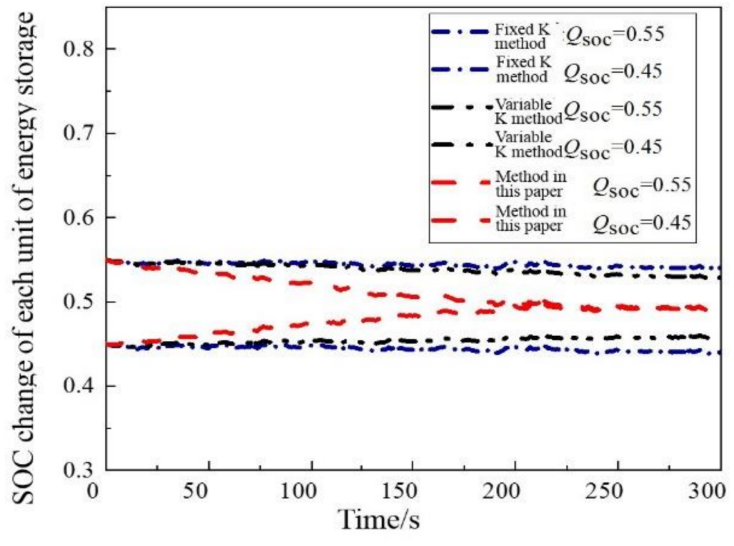

(b)

Figure 8. Case 2: response curve of frequency and SOC: (a) frequency response curve; (b) average SOC change curve. 
Table 2. Case 2: indicator statistics of frequency regulation.

\begin{tabular}{cccc}
\hline Method & $\begin{array}{c}\text { Frequency Index } \\
\boldsymbol{R} \boldsymbol{m} \boldsymbol{s}(f) / \mathbf{1 0}^{-\mathbf{4}}\end{array}$ & $\boldsymbol{R} \boldsymbol{m s}\left(Q_{\text {SOC }}\right)_{\mathbf{1}}$ & $\boldsymbol{R} \boldsymbol{m} \mathbf{s}\left(Q_{\text {SOC }}\right)_{\mathbf{2}}$ \\
\hline No energy storage & 14.567 & $\backslash$ & $\backslash$ \\
Variable K method & 5.678 & 0.0392 & 0.0463 \\
Fixed K method & 3.119 & 0.0450 & 0.0556 \\
Method of this paper & 2.950 & 0.0220 & 0.0260 \\
\hline
\end{tabular}

According to the SOC change curve of each energy storage unit in Figure $8 b$ and the SOC index data in Table 2, under the condition of continuous load disturbance, each unit in the fixed $\mathrm{K}$ method is always assumed to have the same FM responsibility, and the inconsistent SOC of each unit is not improved, which is not conducive to the maximum use of the overall capacity of the energy storage system. In the variable K method, due to the inconsistent initial state of the energy storage unit, the power output of each energy storage unit is carried out according to the values of different optimization curves in the case of charging and discharge. Although the power adjustment of energy storage units in different states is realized to a certain extent, the effect is not obvious, due to the slow change of SOC, and the consistent control of SOC is not achieved until the end of the adjustment cycle. In this paper, the control method SOC has the best maintenance effect. In the process of frequency regulation, the $\mathrm{SOC}$ of each energy storage unit tends to be consistent under the action of equilibrium control, and gradually approaches the reference value of 0.5 . As a result, the two energy storage units in the energy storage system have sufficient bi-directional regulation ability.

\section{Conclusions}

This paper proposes a comprehensive adaptive control strategy for primary frequency modulation of energy storage based on SOC feedback. Specifically, combining the performance advantages of virtual inertia control and droop control, an energy storage primary frequency modulation integrated droop control strategy based on inertia response is constructed. Then, the adaptive output law and balanced control link of the energy storage battery are designed to realize the adaptive adjustment of the output at the level of the energy storage unit and the secondary correction of power coordination at the level of the energy storage system. Finally, the simulation results show that the proposed control strategy can effectively improve the frequency stability and take into account the effect of battery SOC maintenance under different disturbance conditions, such as step and continuous disturbance. In addition, it finely adjusts the output of the energy storage unit, and reasonably coordinates the frequency modulation power of each energy storage unit to achieve SOC equalization control and improve the utilization efficiency of the energy storage system.

Author Contributions: Conceptualization, G.M. and Y.L.; methodology, G.M.; software, Y.L.; validation, Y.Y., Y.S. and W.T.; formal analysis, Y.L.; investigation, G.M.; resources, Y.S.; data curation, Y.Y.; writing—original draft preparation, Y.L.; writing—review and editing, G.M.; visualization, H.L.; supervision, Y.S.; project administration, H.L.; funding acquisition, H.L. All authors have read and agreed to the published version of the manuscript.

Funding: This research was funded by the Natural Science Foundation of the Jiangsu Higher Education Institutions of China, grant number 20KJB480006 and Key Research and Development Program of Jiangsu Province (BE2021094).

Data Availability Statement: No data available.

Conflicts of Interest: The authors declare no conflict of interest. 


\section{References}

1. National Energy Administration Renewable Energy Grid Operation in 2019. 2019, pp. 2-11. Available online: https://djfj. renewable.org.cn/default/coframe/auth/login/login.jsp (accessed on 25 September 2021).

2. Zhang, H.; Sun, Q.; Li, Z.; Bai, Y. Research on cooperative control power generation strategy of wind-hydrogen coupling system. J. Northeast Electr. Power Univ. 2018, 38, 15-23.

3. Zhong, W.; Xie, K.; Liu, Y.; Yang, C.; Xie, S.; Zhang, Y. Online control and near-optimal algorithm for distributed energy storage sharing in smart grid. IEEE Trans. Smart Grid 2019, 11, 2552-2562. [CrossRef]

4. Li, J.; Ma, Y.; Mu, G.; Feng, X.; Yan, G.; Guo, G.; Zhang, T. Optimal configuration of energy storage system coordinating wind turbine to participate power system primary frequency regulation. Energies 2018, 11, 1396. [CrossRef]

5. Liu, J.; Yao, W.; Wen, J.; Fang, J.; Jiang, L.; He, H.; Cheng, S. Impact of power grid strength and PLL parameters on stability of grid-connected DFIG wind farm. IEEE Trans. Sustain. Energy 2020, 1, 545-557. [CrossRef]

6. Shen, G.; Li, S.; Xu, B.; Hui, X.; Song, K. Optimal allocation of energy storage considering wind power integrated to systems. J. Northeast Electr. Power Univ. 2018, 38, 27-34.

7. Bingying, S.; Shuili, Y.; Zongqi, L.; Tingting, L. Analysis on present application of megawatt-scale energy storage in frequency regulation and its enlightenment. Autom. Electr. Power Syst. 2017, 41, 8-16.

8. Li, J.; Wang, S.; Ye, L.; Fang, J. A coordinated dispatch method with pumped-storage and battery-storage for compensating the variation of wind power. Prot. Control Mod. Power Syst. 2018, 3, 21-34. [CrossRef]

9. Li, J.; Feng, X.; Ge, Y.; Li, D.; Fu, Y.; Yan, G. Survey on frequency regulation technology in high wind penetration power system. Power Syst. Prot. Control 2018, 46, 163-170.

10. Zhang, Y.J.A.; Zhao, C.; Tang, W.; Low, S.H. Profit maximizing planning and control of battery energy storage systems for primary frequency control. IEEE Trans. Smart Grid 2018, 9, 712-723. [CrossRef]

11. Li, Y.; He, L.; Liu, F. Flexible voltage control strategy considering distributed energy storages for DC distribution network. IEEE Trans. Smart Grid 2019, 10, 163-172. [CrossRef]

12. Yan, G.; Liu, D.; Li, J.; Mu, G. A cost accounting method of the li-ion battery energy storage system for frequency regulation considering the effect of life degradation. Prot. Control Mod. Power Syst. 2018, 3, 43-51. [CrossRef]

13. Li, R.; Li, X.; Tan, Z.; Huang, J.; He, L. Integrated control strategy considering energy storage battery participating in secondary frequency regulation. Autom. Electr. Power Syst. 2018, 42, 74-82.

14. Almeida, P.R.; Lopes, J.P.; Soares, F.J.; Seca, L. Electric vehicles participating in frequency control: Operating is-landed systems with large penetration of renewable power sources. In Proceedings of the 2011 IEEE Trondheim PowerTech, Trondheim, Norway, 19-23 June 2011; pp. 1-6.

15. Lian, B.; Sims, A.; Yu, D.; Wang, C.; Dunn, R.W. Optimizing LiFePO, battery energy storage systems for frequency response in the UK system. IEEE Trans. Sustain. Energy 2016, 8, 385-394. [CrossRef]

16. Ma, Z.; Li, X.; Tan, Z.; Huang, J.; He, L. Primary frequency modulation control method considering energy storage frequency modulation dead zone. Trans. China Electrotech. Soc. 2019, 34, 2102-2115.

17. Deng, X.; Sun, W.; Xiao, H. Integrated control strategy of battery energy storage system in primary frequency regulation. High Volt. Eng. 2018, 44, 1157-1165.

18. Qingfeng, W.; Xiaofeng, S.; Yanan, W. Distributed control strategy for SOC balancing of distributed energy storage systems in microgrid. Trans. China Electrotech. Soc. 2018, 33, 1247-1256.

19. Stroe, D.I.; Knap, V.; Swierczynski, M.; Stroe, A.I.; Teodorescu, R. Operation of a grid-connected Lithium-Ion battery energy storage system for primary frequency regulation: A battery lifetime perspective. IEEE Trans. Ind. Appl. 2017, 53, 430-438. [CrossRef]

20. Wen, K.; Li, W.; Han, S.; Jin, C.; Zhao, Y. The battery energy storage system considering calendar life participates in the collaborative optimization of primary frequency modulation service configuration capacity and control parameters. High Volt. Eng. 2019, 45, 2185-2193. 